\title{
Methodological aspects of small iron concentrations determination in black yeasts grown in the presence of iron oxide nanoparticles
}

\author{
Grigory Yu. Melnikov ${ }^{1, *}$, Tatyana P. Denisova ${ }^{2}$, Alexander P. Safronov ${ }^{1,3}$, Oleg M. Samatov ${ }^{3}$, Roman T. Khandukhanov ${ }^{2}$, \\ Nikita A. Kulesh ${ }^{1}$, Ricardo Andrade ${ }^{4}$, and Galina V. Kurlyandskaya ${ }^{1,5}$ \\ ${ }^{1}$ Ural Federal University, Mira str., 19, Ekaterinburg, 620002, Russia \\ ${ }^{2}$ Irkutsk State University, Nizhnyaya Embankment St., 6, Irkutsk, 664011, Russia \\ ${ }^{3}$ Institute of Electrophysics, Amundsen str., 106, Ekaterinburg, 620016, Russia \\ ${ }^{4}$ Universidad del País Vasco UPV-EHU, SGIKER, Leioa, 48940, Spain \\ ${ }^{5}$ Universidad del País Vasco UPV-EHU, Depto Electricidad y Electrónica, Leioa,48940, Spain
}

\begin{abstract}
Nonpathogenic Exophiala nigrum (black yeasts) unicelular organisms of the Baikal Lake were used as a model system for determination of small iron concentrations in the samples grown without or with controlled amount of maghemite nanoparticles (MNPs) in nutrient. MNPs were produced by the electrophysical laser target evaporation technique. Electrostatically stabilized suspensions were prepared using sodium citrate solutions in distilled water. We assumed that one maximum permissive dose of ionic iron in water $1 \mathrm{MPD}$ is equal to $0.3 \mathrm{mg} / \mathrm{L}$. For biological experiments Saburo liquid nutrient medium was prepared with iron concentrations of $0,10^{2}, 10^{3}$ and $10^{4}$ MPD. One $\mathrm{ml}$ of E. Nigrum cell suspension was added to Saburo liquid nutrient for 24 hours exposure. Followed by sowing onto a solid agar Saburo for 30 days colonies grows. Biosamples for electron microscopy, magnetic and total reflection X-ray fluorescence spectroscopy measurements were collected simultaneously. We were able to comparatively analyze the trace concentrations of iron in the yeast of the order of $10 \mathrm{ppm}$ for control group and $600 \mathrm{ppm}$ for the group grown in the presence of $10^{4} \mathrm{MPD}$ of iron.
\end{abstract}

\section{Introduction}

Living systems strongly depend on iron in variety of life steps [1-2]. Therefore, precise determination of a small amount of iron in the biological and artificial samples mimicking natural tissues is a challenge. Magnetic nanoparticles (MNPs) can enter occasionally or to be onpurpose injected for therapeutic action into the human body. Magnetite $\left(\mathrm{Fe}_{3} \mathrm{O}_{4}\right)$ and maghemite $\left(\gamma-\mathrm{Fe}_{2} \mathrm{O}_{3}\right)$ are most studied magnetic biocompatible materials [3-4]. An accumulation of MNPs and their degradation products in the tissues/biofluids is a dangerous insufficiently understood health hazard [5]. Nonpathogenic Exophiala nigrum (unicelular black yeast) play an important role in the equilibrium of the Baykal Lake ecosystem [6]. E. Nigrum were previously used as a model system for the estimation of cytotoxicity, MNPs accumulation and inductive heat capacity studies of the samples grown in the presence of MNPs [4,7]. One of the main obstacles for rapid extension of biomedical applications of MNPs is the variation of the properties of MNPs from batch to batch [8]. Therefore, the fabrication techniques providing enhanced batches attract special attention. From this point of view electrophysical techniques of the electric explosion of wire ensuring fabrication of spherical MNPs [9-10] and laser target evaporation (LTE) with the production rates up to $50 \mathrm{~g} / \mathrm{h}$ [11-12] are of special interest. Preparation of model samples with of MNPs is highly requested nowadays for the development of cheap compact analytical devices (magnetic biosensors) [13-15]. They are also requested for better understanding of mechanisms and elaboration of the treatment conditions for such cancer therapies as hyperthermia and thermal ablation [4, 16-17].

In this work, maghemite spherical magnetic nanoparticles were fabricated by LTE technique, and water-based suspensions were prepared on the basis of obtained MNPs. Their physical properties were carefully checked by a number of techniques prior to determination of trace concentrations of iron in the Exophiala nigrum samples grown without or with controlled amount of MNPs in nutrient medium.

\section{Experimental}

Iron oxide MNPs were synthesized by LTE method using Ytterbium $(\mathrm{Yb})$ fiber laser with $1.07 \mu \mathrm{m}$ wavelength. More details can be found elsewhere $[4,7]$. Structural studies of air-dry MNPs were performed by transmission electron microscopy (TEM) using a JEOL

\footnotetext{
* Corresponding author: grisha2207@list.ru
} 
JEM2100 microscope operating at $200 \mathrm{kV}$ and scanning electron microscopy (Hitachi S-4800, SEM). The X-ray diffraction (XRD) studies were performed by DISCOVER D8 (Bruker) diffractometer using $\mathrm{Cu}-\mathrm{K} \alpha$ radiation (wave lengths $\lambda=1.5418 \AA$ ).

Electrostatically stabilized suspensions were prepared by ultrasound treatment using sodium citrate solutions $(5 \mathrm{mM})$ in distilled water. De-aggregation was insured by ultrasound treatment on Cole-Parmer CPX750 homogenizer operated at power output $300 \mathrm{~W}$. During the ultrasound treatment the decrease of an average hydrodynamic diameter of aggregates in suspension was monitored by dynamic light scattering. A Hermle Z383 centrifuge operated at $10,000 \mathrm{rpm}$ for 10 min was used to remove all large aggregates. The specific surface area of MNPs was measured by the lowtemperature sorption of nitrogen (Brunauer-EmmettTeller physical adsorption method, BET) using Micromeritics TriStar 3000 analyzer. More details on suspension preparation can be found elsewhere $[4,11]$.

Maximum permissive dose of iron in water (MPD) is determined as $0.3 \mathrm{mg} / \mathrm{L}$ for ionic iron [18]. The control was Saburo liquid medium with the corresponding addition of a sterile physiological solution without MNPs. As the first step of the biological experiments Saburo liquid nutrient medium was prepared with following iron concentrations (provided by MNPs from suspension): $10^{2}, 10^{3}$ and $10^{4}$ MPD. One $\mathrm{ml}$ of $E$. Nigrum cell suspension was added to Saburo liquid nutrient for 24 hours exposure in flasks using a mechanical rocking chair. At the end of the exposure period sowing onto a solid agar Saburo was done from which biosamples for TEM, magnetic measurements and total reflection X-ray fluorescence (TXRF) measurements were collected simultaneously after 30 days colonies grows. For TEM studies of biological samples cell cultures were pre-fixed in $0.5 \%$ glutaraldehyde in Sörenson buffer and centrifuged. The supernatant was removed and $2 \%$ glutaraldehyde in Sörenson buffer was added to the pellet. Sampleswere fixed in 1\% Osmium Tetroxide in Sörenson buffer, dehydrated, embedded in EponPolarbed resin and cut in ultrathin sections for TEM studies (Philips EM208S). More details on LTE technique can be found elsewhere [15]. TXRF is a method of elemental analysis that can be applied to samples in the form of thin layer. Nanohunter spectrometer by Rigaku and previously elaborated protocol [7] were used. For both scanning electron microscopy (SEM) and TEM of E. Nigrum cell cultures were prepared by standard procedure [15]. Magnetic measurements were carried out on a MPMS XL-7 SQUID-magnetometer in polystyrene envelopes of known mass, contribution of which was carefully subtracted from the biological sample signal. Magnetic measurements were done at room temperature.

\section{Results and discussion}

XRD spectra gave a mean crystallite size for air-dried MNPs of $19 \pm 2 \mathrm{~nm}$ in a good agreement with TEM and specific surface area evaluation. Fig. 1 shows the air-dry
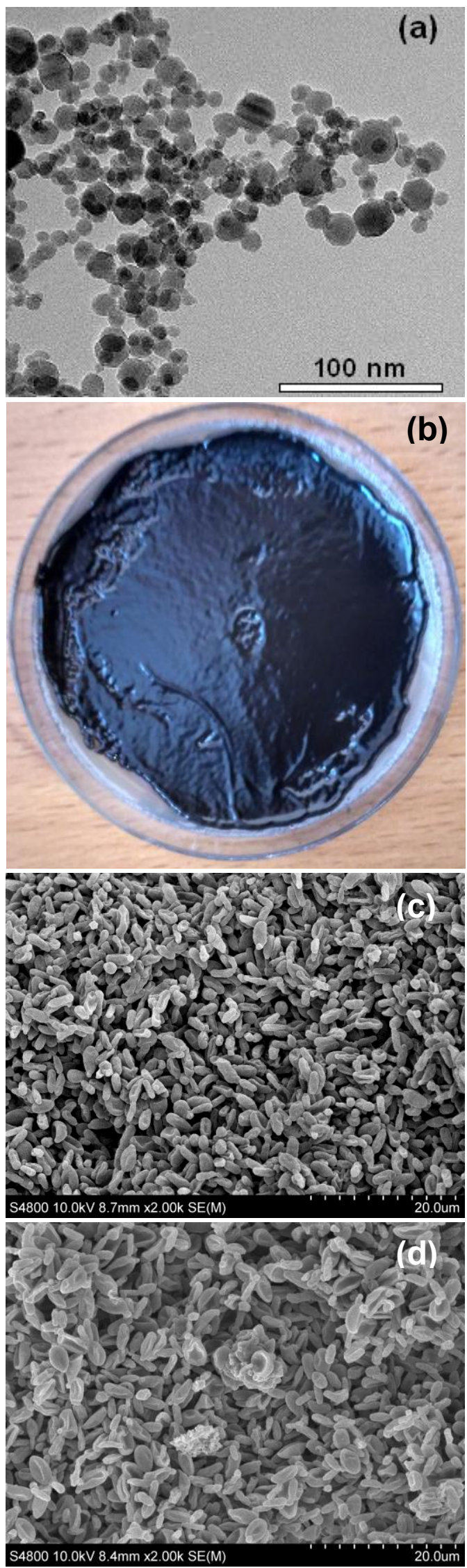

Fig. 1. LTE iron oxide MNPs, TEM (a). Petri dish with E. Nigrim grown onto solid nutrient medium without MNPs (b). General view of E. Nigrum grown in the presence of 0 (c) or $10^{4}$ MPD (d) of iron: MPD of iron provided by MNPs. 
spherical LTE MNPs. The experimental XRD data were well fitted by the magnetite database. The chemical composition of LTE MNPs $\left(\mathrm{Fe}_{2.72} \mathrm{O}_{4}\right)$ close to the stoichiometric maghemite was determined by the combination of red-ox titration and the lattice period analysis provided by XRD as in [9]. We were not able to observe significant difference in the average size/shape of the cells in all cultures under consideration grown in the presence or absence of MNPs (Fig. 1-2, Table 1).
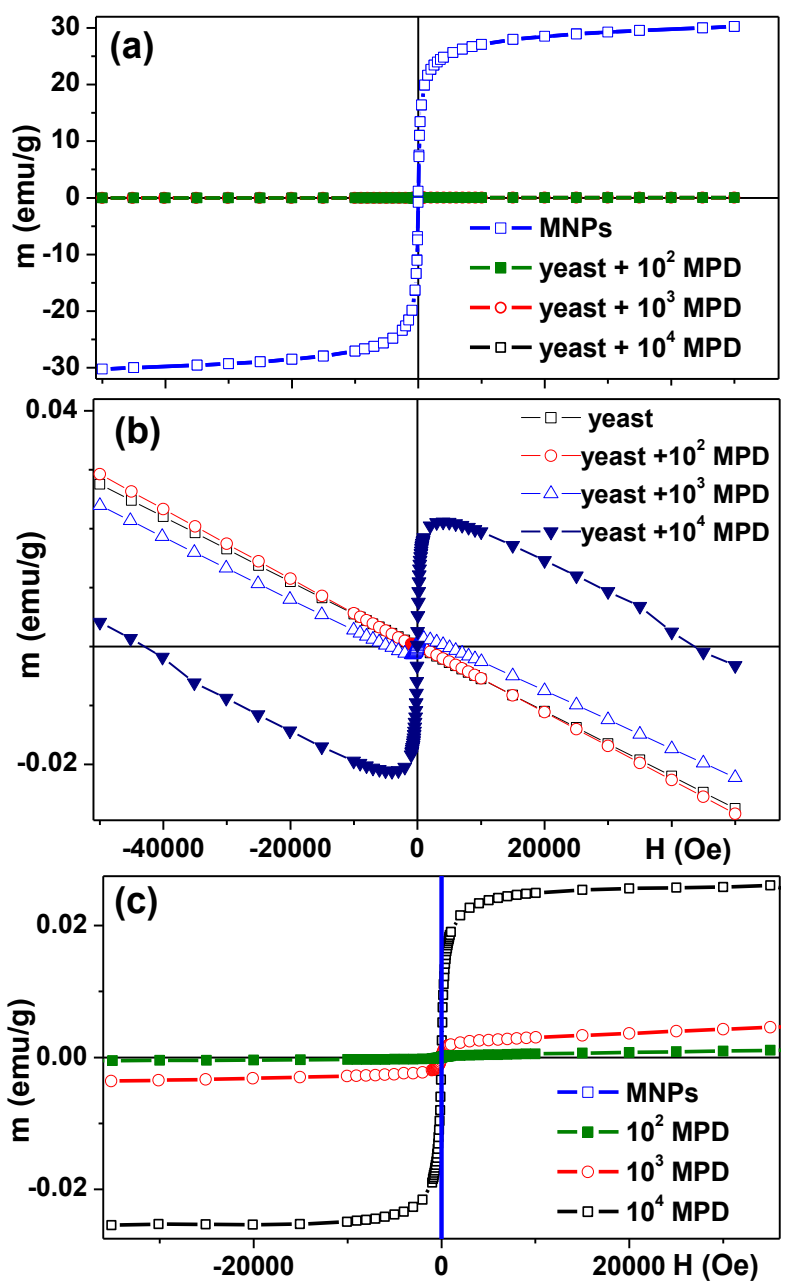

Fig. 2. Hysteresis loops of LTE MNPs from ferrofluid and $E$. Nigrum cell cultures grown in the presence of different amounts of MNPs (a). Hysteresis loops of all cell cultures (b). Recalculated hysteresis loops of all cell cultures grown in the presence of MNPs without contribution of yeast part (c).

Table 1. Iron concentration in Exophiala Nigrum cell cultures grown without or in the presence of MNPs. $\mathrm{M}_{\mathrm{s}}{ }^{*}$ - magnetic moment for $1 \mathrm{~g}$ of the biosample in the field of $35 \mathrm{kOe}$.

\begin{tabular}{|l|l|l|l|}
\hline $\begin{array}{l}\text { MPD in nutrient } \\
\mathrm{mg} / \mathrm{L}\end{array}$ & $\begin{array}{l}\mathrm{M}_{\mathrm{s}}{ }^{*}(\mathrm{emu} / \mathrm{g}) \\
\text { SQUID }\end{array}$ & $\begin{array}{l}\text { ppm Fe by } \\
\text { SQUID }\end{array}$ & $\begin{array}{l}\text { ppm Fe by } \\
\text { TXRF }\end{array}$ \\
\hline 0 & 0.0000 & $0 \pm 10$ & $9 \pm 10$ \\
\hline $10^{2}$ & 0.0009 & $21 \pm 10$ & $15 \pm 10$ \\
\hline $10^{3}$ & 0.0043 & $101 \pm 20$ & $44 \pm 15$ \\
\hline $10^{4}$ & 0.0255 & $603 \pm 100$ & $440 \pm 50$ \\
\hline
\end{tabular}

Fig.2(a) shows results of the SQUID measurements of the air dried MNPs and yeast samples. The yeast samples were taken by sterile scriber and immediately placed into polystyrene envelopes of known mass, mass
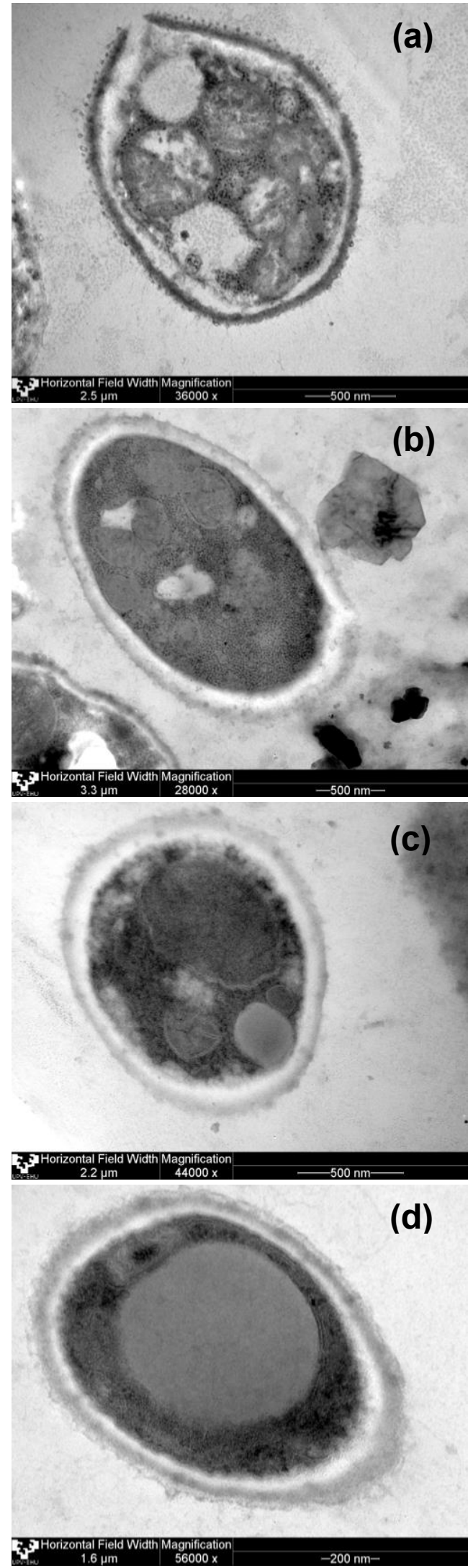

Fig. 3. TEM image of E. Nigrum cells grown without MNPs (a) and with $10^{2}(\mathrm{~b}), 10^{3}(\mathrm{c})$ and $10^{4}$ MPD of iron.

of the yeast was measured. The polysterene contribution was previously measured by SQUID in the same conditions. As to expect, the highest magnetic moment corresponds to the MNPs dried from ferrofluid due to the 
highest MNPs concentration. No saturation at room temperature was observed in the field of $50 \mathrm{kOe}$ which is typical for superparamagnetic samples $[4,14]$. Observed magnetization value is consistent with the mean diameter of $19 \pm 2 \mathrm{~nm}$ for MNPs [7,9]. Although non-zero coercivity $(\approx 50$ Oe at $300 \mathrm{~K}$ ) was observed it can be understood in the frame of the core-shell model invoking a ferromagnetic core and a spin-glass like surface layer in which the spins are frozen without long range magnetic order [11].

Magnetic moment $(\mathrm{m})$ field dependences for all cases had strong diamagnetic contribution as to expect for water containing biosamples (Fig. 2(b)). In order to calculate the magnetic signal corresponding to MNPs per mass unit we used E. Nigrum cell culture grown without MNPs signal for calibration. For each field point we recalculated the hysteresis loops of all samples without diamagnetic contribution (Fig. 2(c). As the saturation was not reached even for high field all calculations were made for the field of $\mathrm{H}=35 \mathrm{kOe}$ wich was reasonably high: $\mathrm{M}_{\mathrm{s}}{ }^{*}$ is a magnetic moment of $1 \mathrm{~g}$ of biosample for the external field $\mathrm{H}=35 \mathrm{kOe}$. Increase of the $\mathrm{M}_{\mathrm{s}}{ }^{*}$ value with MPD increase and " $\mathrm{S}$ " shapes of the hysteresis loops of biosamples after exposure to MNPs indicate MNPs accumulation with the increase of MPD of iron in the initial nutrient. Taking into account parameters of the hysteresis loop for MNPs we were able (Table 1) to recalculate iron concentration in $\mathrm{ppm}$ for SQUD measurements and compare them whith ppm concentrations obtained by TXRF studies of dried samples: the higher MPD in nutrient the higher ppm of iron in the biological samples in both TXRF and SQUID measurements. For recalculation we considered that airdry MNPs magnetic signal $(\mathrm{H}=35 \mathrm{kOe})$ corresponds to $100 \%$ of concentration. Therefore, simple proportion relations with air-dry signal as calibration gave numbers for all concentrations. One can see that SQUID measurements tend to give slightly higher value comparing with TXRF which is surprising because TXRF measures both ionic and MNPs contribution but SQUID signal is mostly corresponds to the MNPs. More studies are necessary in order to clarify the origin of such a discrepancy but it seems to be that E. Nigrum cells are rather large and special improved protocol is necessary for thin film on their basis preparation for TFRF. Trace iron concentrations indicated limited penetration of MNPs inside the cell or their adsorption by cell membranes (Fig. 3 shows some examples). One can clearly see thick cell membrane with typical for $E$. Nigrum morphology. Comparative estimation of the average size of the oval-shaped Exophiala Nigrum cells gives approximate values of $6 \pm 1 \mu \mathrm{m} \times 3 \pm 1 \mu \mathrm{m}$ for SEM and of $5 \pm 1 \mu \mathrm{m} \times 3 \pm 1 \mu \mathrm{m}$ for TEM studies for all cell cultures in a good agreement with each other.

\section{Conclusions}

Nonpathogenic E. Nigrum unicelular yeasts were used as a model system for determination of trace iron concentrations. They were grown in the presence of $19 \pm$ $2 \mathrm{~nm}$ maghemite MNPs produced by laser target
evaporation.Electrostatically stabilized water based suspensions were prepared: liquid nutrient medium was elaborated with different iron concentrations of 0 to $10^{4}$ MPD provided by MNPs. One $\mathrm{ml}$ of E. Nigrum cell suspension was added to Saburo liquid nutrient for 24 hours exposure followed by sowing onto a solid agar Saburo for 30 days cell grows after which the biosamples for TEM, magnetic and TXRF measurements were collected simultaneously. In both TRXRF and SQUID measurements similar tendency was observed: the higher MDP in nutrient the higher ppm of iron in the biological samples. Trace iron concentrations of the order of $10 \mathrm{ppm}$ for control group and $600 \mathrm{ppm}$ for the group grown in the presence of $10^{4}$ MPD of iron provided by MNPs indicated limited penetration of MNPs into the cell or their adsorption by membranes.

The work was supported in part by the Spanish MEC project MAT2014-55049-C2-1-R and RFFI №16-34-50192 grant. We thank Iu.P. Novoselova, I.V. Beketov, E.A. Stepanova, A. Larrañaga and A.S. Volegov for special support. Selected measurements were made at SGIKER services of UPV/EHU and URFU common services.

\section{References}

1. M. Ilberta and V. Bonnefoy, BBA - Bioenerg. 1827, 161-175 (2013).

2. E. Vorburger Mielczarek and Sh. Bertsch McGrayne, Iron, Nature's Universal Element, (Rutgers University Press, 2000).

3. R. C. O'Handley, Modern Magnetic Materials (New York, NY, USA: Wiley, 1972).

4. J.P. Novoselova, A.P. Safronov, O. Samatov, et al., IEEE Trans. Magn. 50, 4600504 (2014).

5. H. Matusiewicz, Acta Biomater.10, 2379-2403 (2014).

6. I.P. Babieva and I.Yu. Chernov, Biology of yeasts (M.V. Lomonosov MSU, Moscow, 2004).

7. N.A. Kulesh, I.P. Novoselova, A.P. Safronov, et al., J. Magn. Magn. Mater. 415, 39-44 (2016).

8. J.H. Grossman and S.E. McNeil, Phys. Today, 65, 3842 (2012).

9. I.V. Beketov, A.P. Safronov, A.I. Medvedev, et al., AIP Adv. 2, 022154 (2012).

10. V. Romanova, G. Ivanenkov, A. Mingaleev, et al., Plasma Phys. Rep. 41, 617-636 (2015).

11. A.P. Safronov, I.V. Beketov, S.V. Komogortsev, et al. , AIP Adv. 3, 052135 (2013).

12. V.V. Osipov, V.V. Platonov, M.A. Uimin, and A.V. Podkin, Tech. Phys. 57, 543-549 (2012).

13. J. Llandro, J.J. Palfreyman, A. Ionescu, et al., Med. Biol. Eng. Comput. 48, 977-998 (2010).

14. G.V. Kurlyandskaya, E. Fernandez, A.P. Safronov, et al., Appl. Phys. Lett. 106, 193702 (2015).

15. G.V. Kurlyandskaya, Iu.P. Novoselova, et al., J. Magn. Magn. Mater. 431, 249-254 (2017).

16. P. Moroz, S.K. Jones, and B.N. Gray, J. Surgical Oncol. 77, 259-269, (2001).

17. M. Coïsson, G. Barrera, F. Celegato, et al., J. Magn. Magn. Mater. 415, 2-7 (2016).

18.https://ohranatruda.ru/ot_biblio/normativ/data_normat iv/9/9742/SanPiN2.1.4.1074-01. 